\title{
Evaluation of the effect of pregabalin on simulated driving ability using a driving simulator in healthy male volunteers
}

This article was published in the following Dove Press journal:

International Journal of General Medicine

29 January 2014

Number of times this article has been viewed

\section{Tomoaki Tujii \\ Win Thiri Kyaw \\ Hirotaka Iwaki \\ Noriko Nishikawa \\ Masahiro Nagai \\ Madoka Kubo \\ Masahiro Nomoto}

Department of Neurology and Clinical Pharmacology, Ehime

University Graduate School of

Medicine, Tohon Ehime, Japan
Correspondence: Masahiro Nomoto

Department of Neurology and Clinical

Pharmacology, Ehime University

Graduate School of Medicine, Tohon,

Ehime 79I-0295, Japan

Tel +8I 899605095

Fax +8I 899605938

Email nomoto@m.ehime-u.ac.jp
Abstract: Pregabalin, a novel agent for treating partial epilepsy and peripheral neuropathic and central pain, was studied for its effect on driving performance in healthy volunteers. Sixteen healthy male volunteers who drove regularly were enrolled in a double-blind, parallel-group, placebocontrolled study assessing the effect of pregabalin on driving performance. Subjects received an oral dose of pregabalin $75 \mathrm{mg}$ or placebo, and a second dose 12 hours later. A driving simulator was used to test simple and complicated braking reaction time, and simple and complicated steering-wheel techniques before the first dose, and 1 hour and 3 hours after the second dose of pregabalin or placebo. The effect of training during the driving test on the driving performance of each group was also evaluated. There were no statistically significant differences in driving performance between the pregabalin and the placebo groups. However, the pregabalin group showed no significant improvement in steering-wheel skills with training, whereas the placebo group showed a significant $(P<0.05)$ improvement with training. In this study using a driving simulator, pregabalin did not impair driving performance but mildly reduced the training effects of driving experiments. Although pregabalin caused sleepiness, it had no severe effect on driving ability after a second dose of $75 \mathrm{mg}$ after the initial introduction of pregabalin.

Keywords: pregabalin, driving, volunteers

\section{Introduction}

Pregabalin, an analog of GABA ( $\gamma$-aminobutyric acid), is an anticonvulsant drug used in the treatment of partial epilepsy. Similar to its parent drug, gabapentin, pregabalin binds to the $\alpha_{2} \delta$ subunit of $\mathrm{Ca}^{2+}$ channels of presynaptic neurons. ${ }^{1}$ This binding reduces the release of neurotransmitters including glutamate, noradrenaline, and substance P, which results in anticonvulsant, anxiolytic, and analgesic effects. ${ }^{2}$

Pregabalin was effective as an add-on therapy for seizure reduction in patients with partial epilepsy with or without secondary generalization in a randomized, doubleblind, placebo-controlled trial. ${ }^{3}$ It was well tolerated, with adverse effects generally reported to be mild-to-moderate somnolence, dizziness, ataxia, diplopia, and weight gain. ${ }^{3}$ Pregabalin has been shown to ameliorate the symptoms of diabetic peripheral neuropathic pain and postherpetic neuralgia. ${ }^{4}$ The sleep quality of these patients was also improved. ${ }^{4}$ A clinical trial of pregabalin has shown a significant improvement in anxiety scores in patients with generalized anxiety disorder. ${ }^{5}$ Pregabalin has been studied in patients with neuropathic pain due to spinal cord injury in a randomized, controlled trial. ${ }^{6}$ There was a significant improvement in endpoint mean pain scores and assessments of sleep. However, dizziness and somnolence were reported as the most frequent adverse effects. 
Although pregabalin is well applied as an effective medication in various indications, the common side effects of drowsiness and dizziness have given rise to questions with respect to safety during driving and handling machinery while the patient is on medication. In this study, we evaluated the effect of pregabalin on the driving performance of healthy volunteers by using a driving simulator to provide more-detailed prescribing information for patients.

\section{Materials and methods}

The study was approved by the Ethical Committee of Ehime University Hospital. Written informed consent was obtained from the volunteers prior to participation in the study, which was performed in accordance with the principles of the Declaration of Helsinki.

Sixteen healthy male volunteers licensed to drive a motor vehicle and with regular driving experience and normal vision were enrolled in this randomized, double-blind, parallelgroup, placebo-controlled study. A crossover design was not employed to exclude learning effects. Exclusion criteria consisted of abnormal complete blood count, infectious diseases, abnormalities on electrocardiogram and thoracic radiography, and use of any medication 72 hours prior to the study. The volunteers received a tablet of either pregabalin 75 mg (Lyrica ${ }^{\circledR}$, Pfizer, Inc, New York, NY, USA) or matching placebo (lactose) on two occasions separated by a 12 hour interval while fasting, ie, a total pregabalin dose of $150 \mathrm{mg}$. This is because the study simulated normal clinical conditions for patients first starting to take pregabalin. Typically, a patient who is prescribed pregabalin by a physician will take a tablet of pregabalin after dinner and will drive his car the next morning after taking a second tablet of pregabalin after breakfast. Driving performance was evaluated using a driving simulator before taking the first dose, and 1 hour and 3 hours after taking the second dose. The rationale for driving assessment at these times was to study correlation with maximum plasma concentrations of pregabalin. Plasma pregabalin concentration, serum creatinine, and estimated glomerular filtration rate (eGFR) were also measured during the study. Physical examination, including vital signs and electrocardiogram tests, were performed before and after the study to detect any side effects of treatment.

\section{Driving simulator}

The volunteers underwent a driving test using a driving simulator (Safety Master NT-932; Niigata Tsushinki Co, Ltd, Niigata, Japan) (Figure 1), which has been adopted officially at drivers' license centers in Japan. They were examined on

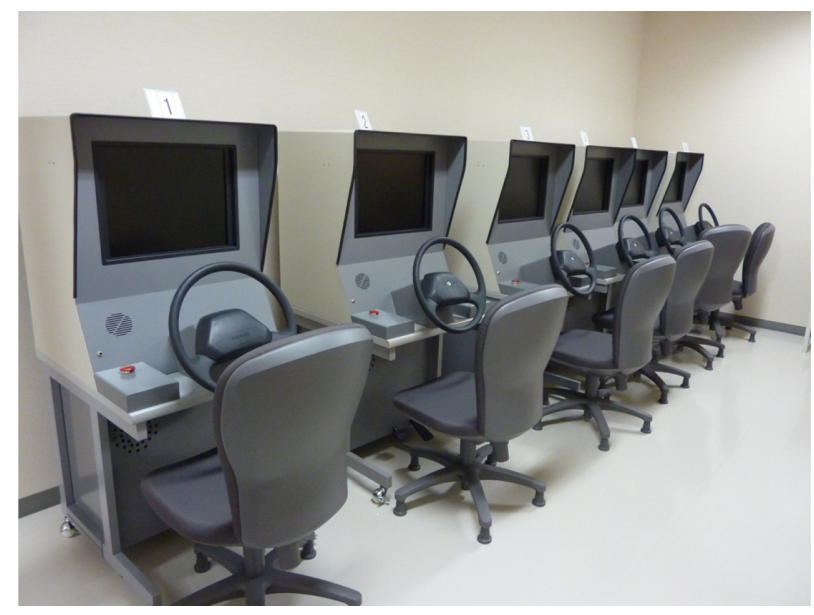

Figure I Driving simulator (Safety Master-NT 932).

their coping responses to a complex of motor ability and perception tasks, using simple reaction time responding to red traffic signals, complicated reaction time responding to variable signals, simple steering-wheel technique responding to variable signals, and steering-wheel technique complicated by braking responding to variable signals. They were required to handle a steering-wheel to avoid collision with a wall and to respond to the change in color of signals on the display by pressing and releasing the accelerator or brake pedal as necessary. The total duration of the test is about 20 minutes and the total scores of the safety driving test are rated on a 5 -point scale ( $1=$ very poor and unsafe, $2=$ moderately poor and unsafe, $3=$ marginal, $4=$ moderately safe, and $5=$ safe driving status) based on the mean result of test items in the driving performance test.

\section{Simple steering-wheel test}

In the simple steering-wheel test, the screen displays horizontal bars on the left and right sides of the road. The subjects are required to avoid collision with the bars on both sides of the road. The mean numbers of steering-wheel errors on both sides was evaluated.

\section{Complicated steering-wheel test}

This test assesses the combination of complicated reaction times and simple steering-wheel tests. The screen of the driving simulator displays bars on both sides of the road, and red, yellow, or green traffic lights on either side of the road. The subjects respond to changes in variable signals by pressing or releasing the pedals, as in the test of complicated reaction time, while trying to not collide with the bars. The mean number of steering-wheel errors on both sides of the road was also evaluated. 


\section{Simple reaction time}

This test evaluates the mean reaction time taken to release the accelerator when the red traffic light is displayed seven times on the screen of the driving simulator. A black traffic signal is displayed throughout the test as an indicator to continue pressing the accelerator. When the black signal is changed to red, the participants are required to release the accelerator and press the brake pedal.

\section{Complicated reaction time}

Individual reaction time taken in responding to variable signals of red, yellow, and green lights was tested in the driving simulator. The participants need to press the accelerator in response to a black light throughout the test and respond to a red light as in the test of simple reaction time. The accelerator needs to be released when the black light changes to yellow and pressed in response to the light's changing to green. The mean of ten traffic light changes is calculated by software in the driving simulator and displayed on screen.

\section{Statistical evaluation}

We evaluated the effect of the pregabalin treatment on driving performance compared to the placebo control group. The effect of training on the driving performance was also assessed in relation to baseline results recorded before taking the first dose. The Mann-Whitney $U$-test was applied to study the level of significance, with $P$-values $<0.05$ considered as statistically significant. Results are expressed as mean values \pm standard deviation. Bonferroni's correction for multiplicity was applied.

\section{Results}

Baseline characteristics were similar for the pregabalin and placebo groups, respectively: age $(26.0 \pm 2.9$ years versus $28.6 \pm 3.5$ years $)$, body weight $(69.0 \pm 4.7 \mathrm{~kg}$ versus $62.2 \pm 4.6 \mathrm{~kg})$, serum creatinine $(0.8 \pm 0.04 \mathrm{mg} / \mathrm{dL}$ versus $0.8 \pm 0.05 \mathrm{mg} / \mathrm{dL})$, and eGFR $(94.1 \pm 5.7 \mathrm{~mL} / \mathrm{minute}$ versus $96.7 \pm 6.5 \mathrm{~mL} /$ minute). Maximum plasma pregabalin concentration was reached at 1 hour in six subjects and at 2 hours in two subjects (Table 1).

Six subjects in the pregabalin group $(100 \%)$ and two in the placebo group (33.3\%) reported feeling sleepy. Two subjects of the pregabalin group presented somnolence. None of the subjects in either group had severe adverse effects.

There was no significant difference in either simple or complicated braking reaction time between the pregabalin and placebo groups (Figure 2A and B). Simple and
Table I Individual plasma pregabalin concentrations at baseline (prior to first dose) and after the second dose

\begin{tabular}{llllll}
\hline $\begin{array}{l}\text { Subject } \\
\text { number }\end{array}$ & \multicolumn{5}{l}{ Plasma pregabalin concentration $(\mu \mathrm{g} / \mathrm{mL})$} \\
\cline { 2 - 6 } & Baseline & I hour & 2 hours & 3 hours & 4 hours \\
\hline $\mathrm{I}$ & $\mathrm{I} .05$ & 1.83 & 4.32 & 3.39 & 3.96 \\
2 & 0.89 & 4.54 & 3.52 & 3.39 & 3.10 \\
3 & 0.99 & 3.85 & 4.06 & 3.21 & 3.06 \\
4 & $\mathrm{I} .30$ & 4.78 & 4.37 & 3.10 & 3.70 \\
5 & $\mathrm{I} .22$ & 5.47 & 3.53 & 3.79 & 3.77 \\
6 & $\mathrm{I} .32$ & 4.60 & 3.94 & 3.77 & 3.23 \\
7 & $\mathrm{I} .1 \mathrm{I}$ & 5.78 & 3.71 & 3.19 & 3.01 \\
8 & 0.86 & 4.38 & 3.87 & 3.20 & 3.39 \\
\hline
\end{tabular}
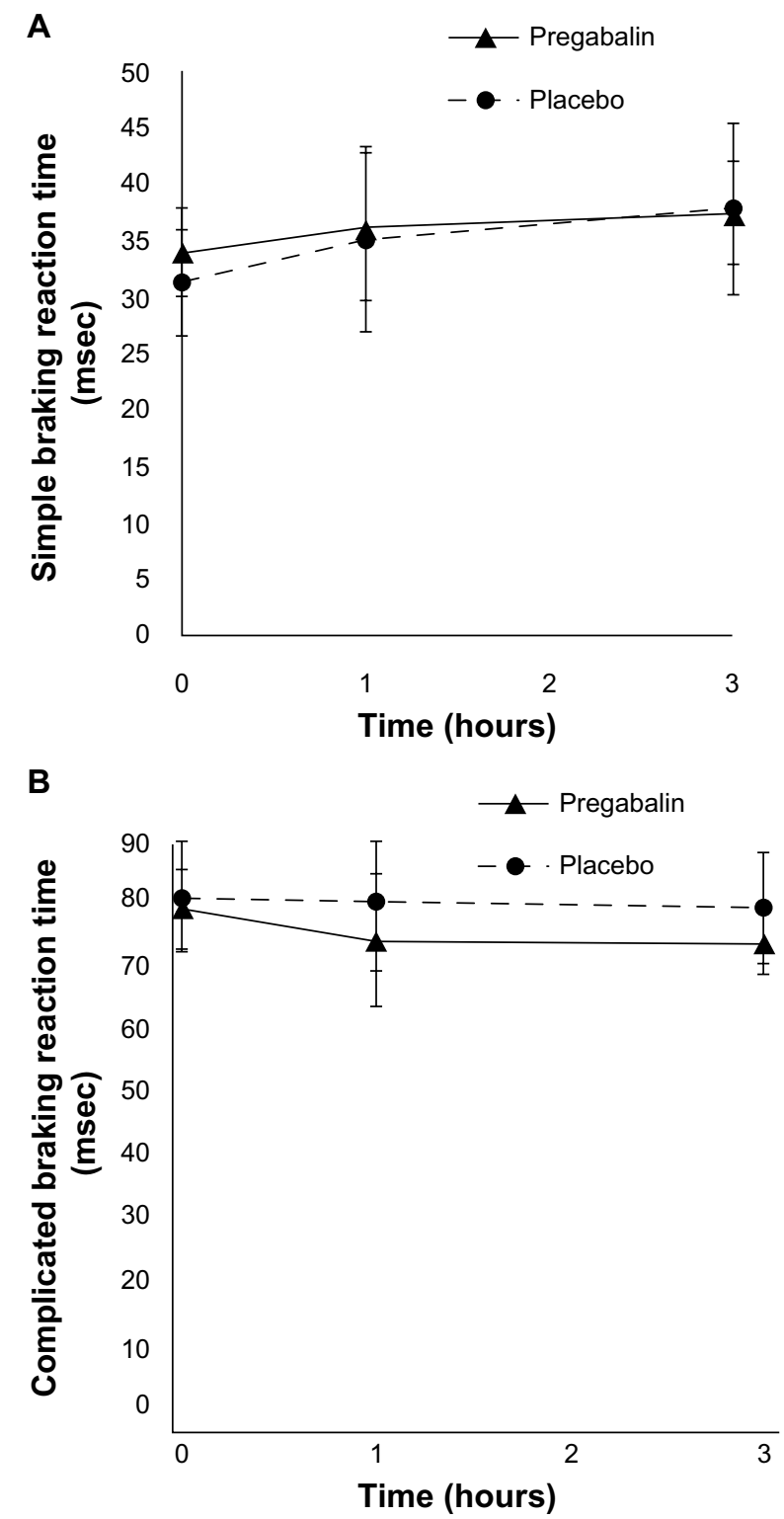

Figure 2 Effect of pregabalin on braking reaction time.

Notes: (A) Simple braking test: reaction time in response to a red light. (B) Complicated braking test: reaction time in response to variable signals. Results from both tests were unaffected by either pregabalin or placebo compared to baseline. There were also no significant changes in both simple and complicated braking reaction time between the pregabalin and placebo groups. 
complicated steering-wheel techniques also showed no significant differences between the pregabalin and placebo groups (Figure 3A and B). When comparing the effect of training on driving performance, the placebo group showed an improvement in simple steering-wheel technique (Table 2; Figure 3A): the number of errors in steering-wheel handling was significantly reduced at 1 hour and 3 hours compared to baseline. However, the pregabalin group showed no improvement in steering-wheel handling with training. There were no differences between the groups or changes compared with baseline in simple and complicated braking reaction times, complicated steering-wheel handling tests, or total safety score comparing pregabalin and placebo (Figure 4).
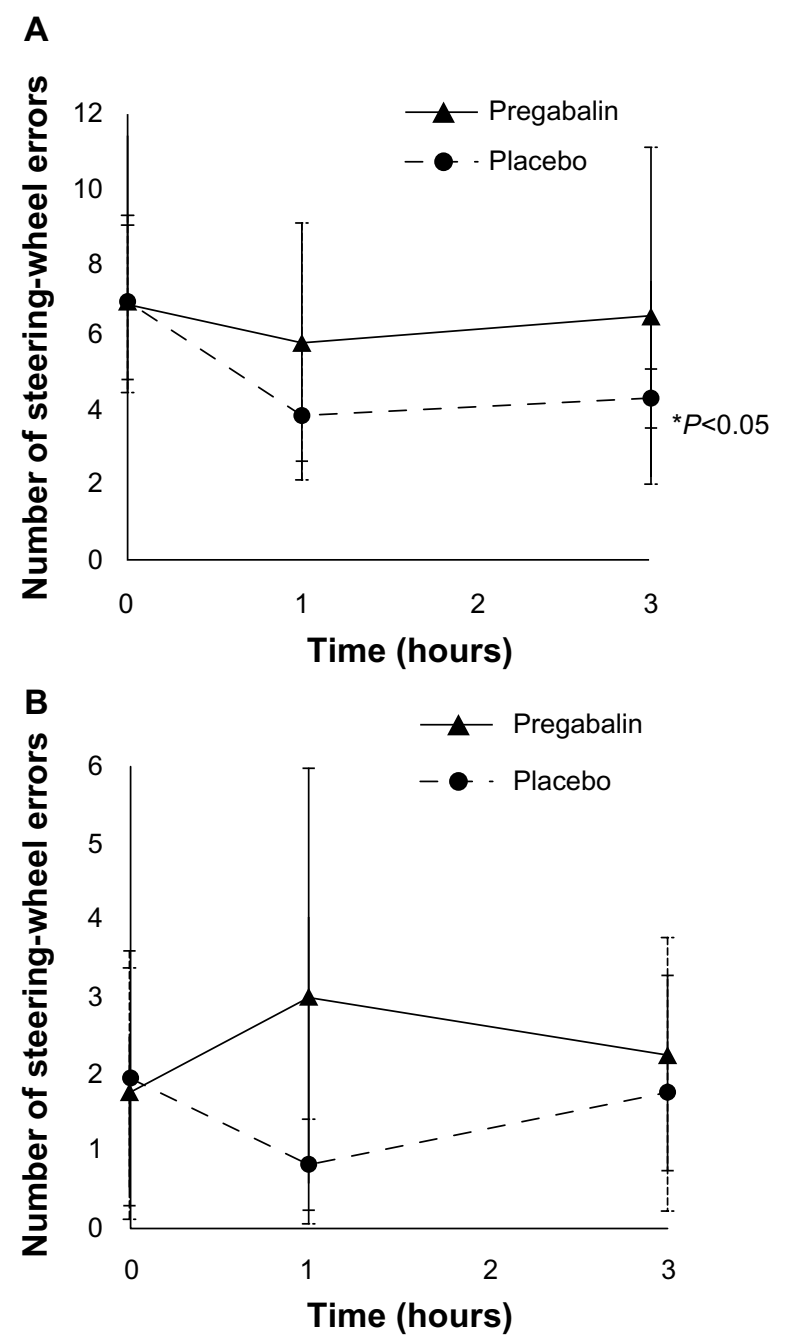

Figure 3 Effect of pregabalin on steering-wheel technique.

Notes: (A) Simple steering-wheel test: The number of simple steering-wheel errors evaluated at $I$ and 3 hours was significantly $(P<0.05)$ decreased compared to baseline in the placebo group, whereas the pregabalin group showed no significant change in the number of simple steering-wheel errors. (B) Complicated steeringwheel test: Both the pregabalin and the placebo groups exhibited no changes in complicated steering-wheel technique under variable signals.
Table 2 Effect of training on driving performance

\begin{tabular}{|c|c|c|}
\hline \multirow[t]{2}{*}{ Driving simulator test } & \multicolumn{2}{|l|}{$P$-value* } \\
\hline & $\begin{array}{l}\text { Baseline } \\
\text { versus } \\
\text { I hour }\end{array}$ & $\begin{array}{l}\text { Baseline } \\
\text { versus } \\
3 \text { hours }\end{array}$ \\
\hline \multicolumn{3}{|l|}{ Pregabalin group $(n=8)$} \\
\hline Simple braking reaction time & 0.79 & 0.49 \\
\hline Complicated braking reaction time & 0.67 & 0.71 \\
\hline Number of errors in simple steering-wheel test & 0.22 & 0.42 \\
\hline $\begin{array}{l}\text { Number of errors in complicated steering- } \\
\text { wheel test }\end{array}$ & 0.42 & 0.34 \\
\hline \multicolumn{3}{|l|}{ Placebo group $(n=8)$} \\
\hline Simple braking reaction time & 0.23 & 0.42 \\
\hline Complicated braking reaction time & 0.56 & 0.40 \\
\hline Number of errors in simple steering-wheel test & $0.01^{\dagger}$ & $0.01^{+}$ \\
\hline $\begin{array}{l}\text { Number of errors in complicated steering- } \\
\text { wheel test }\end{array}$ & 0.10 & 0.91 \\
\hline
\end{tabular}

Notes: *Comparing baseline prior to first dose versus I hour or 3 hours after the second dose; 'according to Bonferroni's correction, $P=0.01$ was estimated to be $P=0.04$.

Abbreviation: $\mathrm{n}$, number.

\section{Discussion}

Central nervous system-depressant drugs increase the risk of traffic accidents ${ }^{7}$ and are assumed to trigger acute impairment during driving. ${ }^{8}$ Benzodiazepines, which are commonly prescribed antidepressant, anticonvulsant, and anesthetic drugs, have been particularly studied for their effect on collisions and driving performance. Benzodiazepines act at the level of the benzodiazepine receptor and enhance the effect of GABA, which causes somnolence in some individuals. ${ }^{7}$

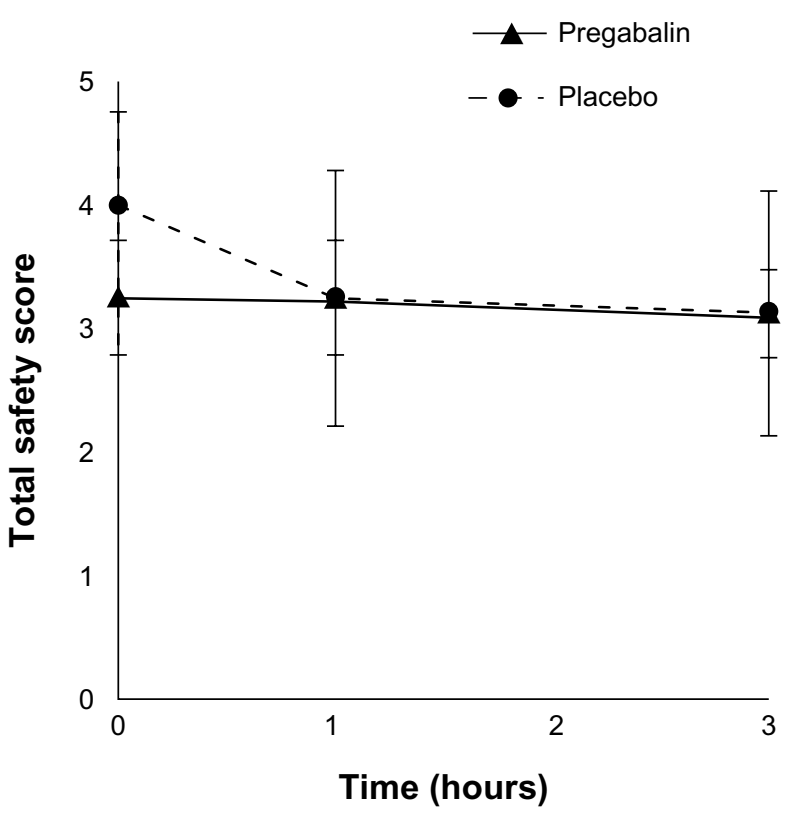

Figure 4 Effect of pregabalin on total safety test scores.

Note: Neither the pregabalin group nor the placebo group showed significant correlation with total safety test scores. 
An epidemiological study has reported that collision risk is associated with long-acting but not short-acting benzodiazepines. ${ }^{9}$ On-road crossover studies assessing the impact of benzodiazepines on driving among healthy subjects ${ }^{10}$ or anxious patients ${ }^{11}$ have also reported no impact on braking reaction time. A review study of real-life driving presented four cases with a positive relationship between plasma gabapentin concentration $(2.0-15.5 \mathrm{mg} / \mathrm{L})$ and impaired driving ability. ${ }^{12}$ These subjects were reported for improper use of turn signal, slow speeds, and deviation from regular driving and braking.

Epidemiological studies of driving in the elderly receiving $\mathrm{H}_{1}$-antihistamines have shown no significant association with car crashes. ${ }^{13,14}$ In contrast, a study conducted among professional drivers reported an increase risk of car crashes with the prescription of antihistamines..$^{15}$ Drinking coffee is assumed to counteract the effect of sleepiness during driving. A simulated highway driving study proved that caffeinated coffee had a positive effect on driving performance and subjective sleepiness, compared to decaffeinated coffee. ${ }^{16}$

Patients with Parkinson's disease (PD) have been reported to have impaired driving performance. ${ }^{17}$ Compared to healthy controls, PD patients made more safety errors both under baseline conditions and during distraction. ${ }^{18}$ Motor assessments alone may not be enough to predict driving performance in PD patients. In a previous study of driving in PD patients using a driving simulator, ${ }^{19}$ we found that patients with more impairment made more steering errors but showed no impairment of braking reaction time. ${ }^{19}$

In the present study, the use of pregabalin was not associated with braking reaction time, although some participants complained of sleepiness during the test. The sample size in this study was limited because of the number of available driving simulators and the time needed to perform the driving test, so further studies are needed. This finding is, however, compatible with other findings on the effect of pregabalin on braking reaction time. In a double-blind study among healthy volunteers ${ }^{20}$ pregabalin did not significantly impair braking reaction time compared to placebo, whereas a positive control with alprazolam caused significant impairment of breaking reaction time. However, pregabalin showed an influence on driving performance in our study, as steering-wheel handling skills were not improved with training whereas the placebo group showed a significant improvement of handling skills with training. Compared to braking reaction time, steeringwheel handling skills seem to be more sensitive to the effect of drugs. In our study, a dose of pregabalin $75 \mathrm{mg}$ was used because we wanted to study the actual outpatient clinical condition - a dose of $75 \mathrm{mg}$ is the recommended dose at the start of treatment. Our study might have some limitations due to the involvement of only young male participants and not including the highest approved dose of pregabalin. The driving simulator provides a comprehensive evaluation of driving, including perception or motor performance. Another recent study of pregabalin did not reveal any interference with psychomotor testing. ${ }^{21}$ Further studies are needed to clarify the effects of pregabalin on driving.

In conclusion, pregabalin showed no severe central nervous system effects related to cognitive measures during simulated driving testing in healthy subjects. Because pregabalin may cause somnolence, on first prescription of the drug we advise patients to be careful when driving, rather than advising them to avoid driving a car totally.

\section{Acknowledgments}

The work was supported by Grants-in-Aid from the Research Committee for Parkinson's Disease, the Ministry of Health, Labour and Welfare of Japan, and SRFJ; and a Grant from Ehime University. The authors would like to express their thanks to all the clinical research coordinators of the Clinical Research Trial Centre of Ehime University Hospital.

\section{Disclosure}

The authors report no conflicts of interest in this work.

\section{References}

1. Gee NS, Brown JP, Dissanayake VU, Offord J, Thurlow R, Woodruff GN. The novel anticonvulsant drug, gabapentin (Neurontin), binds to the alpha2delta subunit of a calcium channel. J Biol Chem. 1996;271(10):5768-5776.

2. Dooley DJ, Mieske CA, Borosky SA. Inhibition of $\mathrm{K}(+)$-evoked glutamate release from rat neocortical and hippocampal slices by gabapentin. Neurosci Lett. 2000;280(2):107-110.

3. Arroyo S, Anhut H, Kugler AR, et al; Pregabalin 1008-011 International Study Group. Pregabalin add-on treatment: a randomized, double-blind, placebo-controlled, dose-response study in adults with partial seizures. Epilepsia. 2004;45(1):20-27.

4. van Seventer R, Bach FW, Toth CC, et al. Pregabalin in the treatment of post-traumatic peripheral neuropathic pain: a randomized double-blind trial. Eur J Neurol. 2010;17(8):1082-1089.

5. Pande AC, Crockatt JG, Feltner DE, et al. Pregabalin in generalized anxiety disorder: a placebo-controlled trial. Am J Psychiatry. 2003;160(3): 533-540.

6. Cardenas DD, Nieshoff EC, Suda K, et al. A randomized trial of pregabalin in patients with neuropathic pain due to spinal cord injury. Neurology. 2013;80(6):533-539.

7. Dassanayake T, Michie P, Carter G, Jones A. Effects of benzodiazepines, antidepressants and opioids on driving: a systematic review and meta-analysis of epidemiological and experimental evidence. Drug Saf. 2011;34(2):125-156.

8. Vermeeren A. Residual effects of hypnotics: epidemiology and clinical implications. CNS Drugs. 2004;18(5):297-328. 
9. Hemmelgarn B, Suissa S, Huang A, Boivin JF, Pinard G. Benzodiazepine use and the risk of motor vehicle crash in the elderly. JAMA. 1997;278(1):27-31.

10. Harrison C, Subhan Z, Hindmarch I. Residual effects of zopiclone and benzodiazepine hypnotics on psychomotor performance related to car driving. Drugs Exp Clin Res. 1985;11(12):823-829.

11. Moore NC. Medazepam and the driving ability of anxious patients. Psychopharmacology (Berl). 1977;52(1):103-106.

12. Peterson BL. Prevalence of gabapentin in impaired driving cases in Washington State in 2003-2007. J Anal Toxicol. 2009;33(8): 545-549.

13. Leveille SG, Buchner DM, Koepsell TD, McCloskey LW, Wolf ME, Wagner EH. Psychoactive medications and injurious motor vehicle collisions involving older drivers. Epidemiology. 1994;5(6):591-598.

14. Ray WA, Fought RL, Decker MD. Psychoactive drugs and the risk of injurious motor vehicle crashes in elderly drivers. Am J Epidemiol. 1992;136(7):873-883.

15. Howard ME, Desai AV, Grunstein RR, et al. Sleepiness, sleepdisordered breathing, and accident risk factors in commercial vehicle drivers. Am J Respir Crit Care Med. 2004;170(9):1014-1021.
16. Mets M, Baas D, van Boven I, Olivier B, Verster J. Effects of coffee on driving performance during prolonged simulated highway driving. Psychopharmacology (Berl). 2012;222(2):337-342.

17. Worringham CJ, Wood JM, Kerr GK, Silburn PA. Predictors of driving assessment outcome in Parkinson's disease. Mov Disord. 2006;21(2):230-235.

18. Uc EY, Rizzo M, Anderson SW, Sparks JD, Rodnitzky RL, Dawson JD. Driving with distraction in Parkinson disease. Neurology. 2006;67(10): 1774-1780.

19. Thiri Kyaw W, Nishikawa N, Moritoyo T, Tsujii T, Iwaki H, Nomoto M. Evaluating the driving ability in patients with Parkinson's disease using a driving simulator. Intern Med. 2013;52(8):871-876.

20. Hindmarch I, Trick L, Ridout F. A double-blind, placebo- and positive-internal-controlled (alprazolam) investigation of the cognitive and psychomotor profile of pregabalin in healthy volunteers. Psychopharmacology (Berl). 2005;183(2):133-143.

21. Zacny JP, Paice JA, Coalson DW. Subjective, psychomotor, and physiological effects of pregabalin alone and in combination with oxycodone in healthy volunteers. Pharmacol Biochem Behav. 2012;100(3): $560-565$.
International Journal of General Medicine

\section{Publish your work in this journal}

The International Journal of General Medicine is an international, peer-reviewed open-access journal that focuses on general and internal medicine, pathogenesis, epidemiology, diagnosis, monitoring and treatment protocols. The journal is characterized by the rapid reporting of reviews, original research and clinical studies across all disease areas.

\section{Dovepress}

A key focus is the elucidation of disease processes and management protocols resulting in improved outcomes for the patient.The manuscript management system is completely online and includes a very quick and fair peer-review system. Visit http://www.dovepress.com/ testimonials.php to read real quotes from published authors. 